\title{
The Hilbert Transform in Analysis of Uterine Contraction Activity
}

\author{
Marta Borowska $^{1}$, Ewelina Brzozowska ${ }^{1}$, Edward Oczeretko $^{1}$
}

1 Department of Materials and Biomedical Engineering, Faculty of Mechanical Engineering, Bialystok University of Technology, Poland

\begin{abstract}
Prevention and early diagnosis of forthcoming preterm labor is of vital importance in preventing child mortality. To date, our understanding of the coordination of uterine contractions is incomplete. Among the many methods of recording uterine contractility, electrohysterography (EHG) - the recording of changes in electrical potential associated with contraction of the uterine muscle, seems to be the most important from a diagnostic point of view. There is some controversy regarding whether EHG may identify patients with a high risk of preterm delivery. There is a need to check various digital signal processing techniques to describe the recorded signals. The study of synchronization of multivariate signals is important from both a theoretical and a practical point of view. Application of the Hilbert transformation seems very promising.
\end{abstract}

\section{Introduction}

A biomedical signal can be described as the reflection or a "shadow" of the process that causes it. By examining the signal, we receive information about the process. Biomedical signals are predominantly characterized by a high degree of complexity; they are far from stationary. The study of synchronization of bivariate biomedical signals is important from both a theoretical and a practical point of view. A change of synchronization parameter can be very useful for medical diagnostics.

The human uterus is undoubtedly a complex system, like the brain or the heart. Billions of myometrium cells interact in a complex manner. Our understanding of the coordination of uterine contractions is incomplete. The myometrial cells can be excited by action potentials generated by their own impulses. There are pacemaker cells, which synchronize the activity of the whole uterus. The other cells are excited by impulses from the neighbouring cells - these are the pacefollower cells. Unlike cardiac cells, the myometrial 
cells can play the roles of pacemaker cells and pacefollower cells alternately. It has been shown in earlier studies that nonlinear processes probably generate uterine contractions (Nagarajan et al., 2003; Oczeretko et al., 2005; Radhakrishnan et al., 2000a).

EHG is a multi-channel measurement method, so it is important to the study of synchronization of signals. The notion of synchronization may be used in a loose sense as the synonym of correlation, the similarity of the signals, or the similarity of their dynamics. The Hilbert transform, the cross-correlation, the coherence, the mutual correlation dimension, the cross-approximate entropy, the mutual information and the nonlinear interdependencies can be applied for this purpose.

For this study, all software implementations were done in Matlab (The MathWorks Inc., Natick, MA).

\section{Hilbert Transform}

The simple and inverse Hilbert transforms are defined by the integrals:

$$
\begin{gathered}
\hat{x}(t)=H[x(t)]=\frac{1}{\pi} \int_{-\infty}^{\infty} \frac{x(\tau)}{t-\tau} d \tau \\
x(t)=H^{-1}[\hat{x}(t)]=-\frac{1}{\pi} \int_{-\infty}^{\infty} \frac{\hat{x}(\tau)}{t-\tau} d \tau
\end{gathered}
$$

These integrals are understood in the sense of the Cauchy principal value, because for $t=\tau$, the integrands in the above formulas are endless. The name "transformation" is used to the right-hand sides of these equations and the name "transform" to the left hand sides (Hahn, 1996). Operator $H$ is a linear operator, i.e., if $a$ and $b$ are arbitrary (complex) scalars, and $x(t)$ and $y(t)$ are signals, then:

$$
H[a x(t)+b y(t)]=a \hat{x}(t)+b \hat{y}(t)
$$

In terms of the convolution notation, the equations defining Hilbert transforms may be written:

$$
\begin{gathered}
\hat{x}(t)=\frac{1}{\pi t} \cdot x(t) \\
x(t)=-\frac{1}{\pi t} \cdot \hat{x}(t)
\end{gathered}
$$


since, for example, using the definition of convolution for the first of the above two equations, it can be presented as:

$$
\hat{x}(t)=\frac{1}{\pi t} \cdot x(t)=\frac{1}{\pi} \int_{-\infty}^{\infty} \frac{x(\tau)}{t-\tau} d \tau=\frac{1}{\pi} \int_{-\infty}^{\infty} \frac{x(t-\tau)}{\tau} d \tau
$$

Applying the Fourier transform to the equation defining the simple Hilbert transform, and using the convolution theorem (the Fourier transform of the convolution is the product of the two Fourier transforms), we get:

$$
F[\hat{x}(t)]=F\left[\frac{1}{\pi t}\right] F[x(t)]
$$

$F$ denoting the Fourier transform operator.

Seeing that (Toland, 1997):

$$
F\left[\frac{1}{\pi t}\right](\omega)=-i \operatorname{sgn}(\omega)
$$

where $\operatorname{sgn}(\omega)$ is the sign function, $\omega$ is the angular frequency or angular speed (measured in radians per second),

$$
\operatorname{sgn}(\omega)= \begin{cases}-1 & \text { for } \omega<0 \\ 1 & \text { for } \omega \geq 0\end{cases}
$$

so:

$$
F[\hat{x}(t)](\omega)=-i \operatorname{sgn}(\omega) \cdot F[x(t)](\omega)
$$

The Hilbert transform can be obtained by multiplying the Fourier spectrum of $x(t)$ signal by $i(+90)$ for negative frequencies and $-i(-90)$ for positive frequencies. Therefore, the Hilbert transform represents a harmonic conjugate of $x(t)$ signal (Sahoo et al., 2011). Using this, the Hilbert transform can be computed in three steps:

- calculation of the Fourier transform of the given signal,

- rejection of the negative frequencies,

- calculation of the inverse Fourier transform

$$
\hat{x}(t)=F^{-1}[-i \operatorname{sgn}(\omega) \cdot F[x(t)](\omega)]
$$

The Hilbert transform is associated with a phase shift of the analyzed signal by 90 degrees. Therefore, a sine signal becomes a cosine signal and vice versa. Below are some examples of Hilbert transformation pairs (pairs, because the signals are on the left sides of the equations and their transformations are on the right). 
Marta Borowska et al.

$$
\begin{gathered}
\sin \omega_{0} t \leftrightarrow-\cos \omega_{0} t \\
\cos \omega_{0} t \leftrightarrow \sin \omega_{0} t \\
\delta(t) \leftrightarrow \frac{1}{\pi t} \\
\operatorname{rect}(t) \leftrightarrow \frac{1}{\pi} \ln \left|\frac{t+\frac{1}{2}}{t-\frac{1}{2}}\right|
\end{gathered}
$$

Applications of the Hilbert transform in the analysis of biomedical signals are connected with the concepts of the analytic signal, envelope, instantaneous phase and instantaneous frequency (Boashash, 1992a, 1992b). Frequency is the number of events (cycles or vibrations) occurring per time unit. Let us consider a material point moving with uniform motion in a circle. Projection of uniform circular motion upon a diameter (Figure 1a) performs the simple harmonic motion:

$$
x(t)=A_{0} \cos \varphi=A_{0} \cos \omega_{0} t
$$

where:

$\varphi$ is the phase,

$A_{0}$ is the amplitude.

The Fourier transformation of the signal is represented by the sum (with the appropriate weights) of sine and cosine functions. The frequency, the amplitude and the initial phase of the component functions are constant. The real signals received by experiments or by routine medical diagnostics are often non-stationary. Such signals are very badly decomposed into harmonic components. Here, the frequency and the amplitude change, so it is necessary to generalize the concept of frequency.

The concept of the analytical signal was presented by Gabor (1946) in optics. For a scalar time series $x(t)$, received from a measurement of a nonlinear system, the corresponding analytic signal $z(t)$ is defined by:

$$
z(t)=x(t)+i H[x(t)]=x(t)+i \hat{x}(t)
$$

where $H[x(t)]$ is the Hilbert transform operator.

The envelope (Figure 1b) (or instantaneous amplitude) of signal $x(t)$ is defined by:

$$
A(t)=\sqrt{x(t) \cdot x(t)+\hat{x}(t) \cdot \hat{x}(t)}
$$


and its instantaneous phase angle (Figure 1b) in the complex plane is

$$
\varphi(t)=\arctan \left\{\frac{\hat{x}(t)}{x(t)}\right\}
$$

Figure 2 shows the envelope of signal $y=3 e^{-4 t} \sin (40 \pi t)$. The following is a Matlab script for finding that envelope.

$\mathrm{t}=0: 1 / 999: 1$

$\mathrm{x}=3^{*} \exp (-4 * \mathrm{t}) \cdot{ }^{*} \sin \left(2^{*} \mathrm{pi}^{*} 20^{*} \mathrm{t}\right)$

$\operatorname{set}\left(0,{ }^{\prime}\right.$ DefaultFigureColor', $\left.\left[\begin{array}{lll}1 & 1 & 1\end{array}\right]\right)$;

plot(x,'Color','k','LineWidth',2);

hold on;

$\mathrm{y}=\operatorname{hilbert}(\mathrm{x})$;

$\mathrm{A}=\operatorname{sqrt}\left(\left(\operatorname{real}(\mathrm{y}) \cdot *^{\mathrm{real}}(\mathrm{y})\right)+\left(\operatorname{imag}(\mathrm{y}) \cdot *^{\mathrm{imag}}(\mathrm{y})\right)\right) ;$

$\mathrm{A}=\operatorname{abs}(\mathrm{y})$;

$\operatorname{plot}\left(\mathrm{A},{ }^{\prime}\right.$ Color', 'k','LineWidth',3);

hold off;
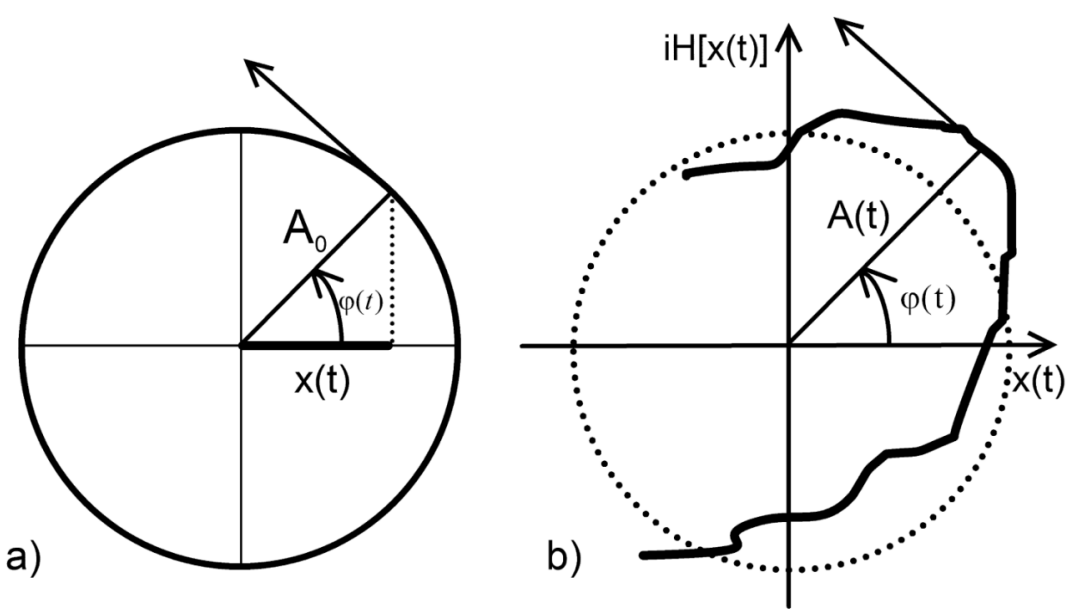

Figure 1. a) Simple harmonic motion $x(t)=A_{0} \cos \varphi=A_{0} \cos \omega_{0} t$, b) Envelope $A(t)$ and instantaneous phase $\varphi(t)$ of signal $x(t)=A \cos \varphi(t)$ (modified from Boashash, 1992a)

The instantaneous frequency is related to the rate of change of the instantaneous phase:

$$
f(t)=\frac{1}{2 \pi} \frac{d \varphi(t)}{d t}
$$

because

$$
\omega=\varphi^{\prime}=2 \pi f
$$


The calculation of the instantaneous frequency for a given signal seems very simple at first glance. In Matlab, there is a "hilbert" function that, when applied to the signal $x$, gives us the analytical signal $y=x+i \hat{x}$. However, the use of the instruction:

$$
\mathrm{f}=\operatorname{diff}(\operatorname{angle}(\operatorname{hilbert}(\mathrm{x})))
$$

where the function angle returns the phase angles in radians, which lie between $-\pi$ and $\pi$, and $\operatorname{diff}(\mathrm{x})$ calculates differences between adjacent elements, leads to unexpected results. Even for sinusoidal signals, we obtain many large spikes in the negative direction. The cause of this is the noise which is amplified by the diff operation. Therefore, as suggested on the website of Matlab (MATLAB Newsgroup, 1997), this operation must be carried out within the function angle, as it can be seen in the following statement:

$$
\mathrm{f}=\operatorname{angle}\left(\operatorname{conv}\left(\operatorname{ones}(1,5), \mathrm{y}(2: \operatorname{length}(\mathrm{y})) \cdot{ }^{*} \operatorname{conj}(\mathrm{y}(1: \operatorname{length}(\mathrm{y})-1))\right)\right)
$$

As a result of using this method, for a signal with several frequencies you will get only the maximum frequency, which we take for the instantaneous frequency value.

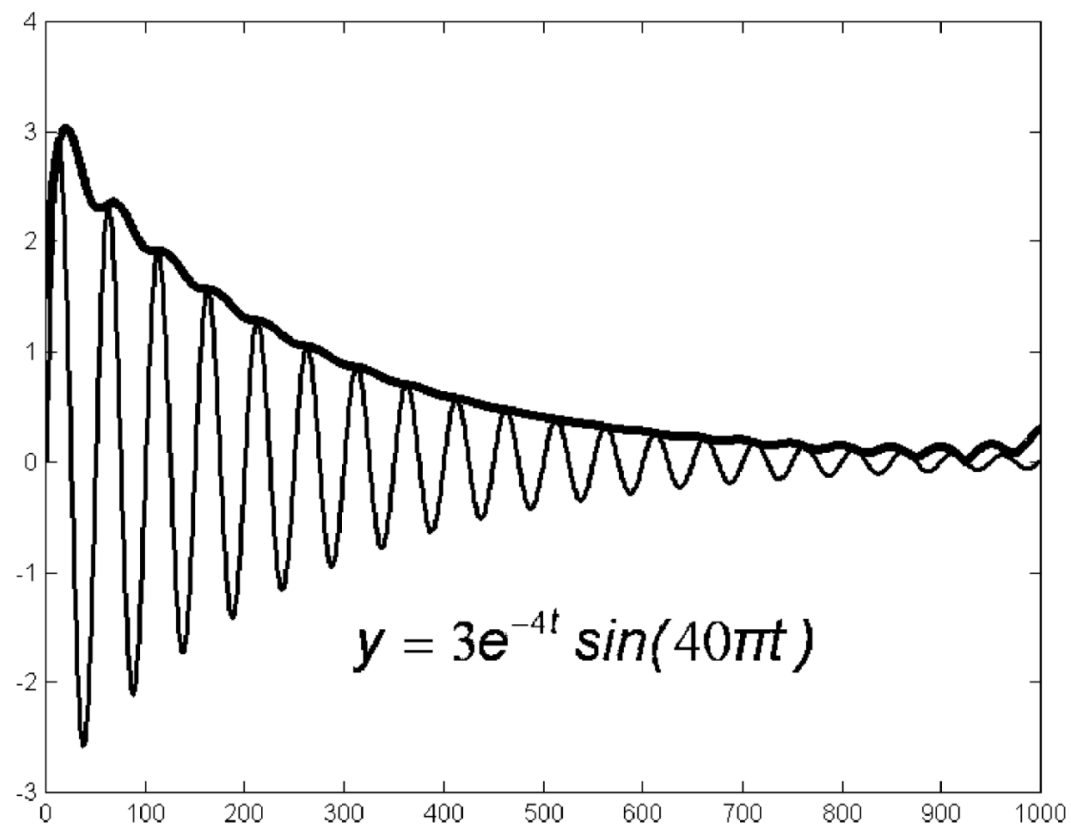

Figure 2. The envelope of signal $y=3 e^{-4 t} \sin (40 \pi t)$ 
Using the Hilbert transform, we can estimate the indicator of phase synchronization from the difference of phases. Systems are synchronized in a phase if:

$$
\varphi_{x, y}(t)=\left|n \varphi_{x}(t)-m \varphi_{y}(t)\right| \leq \text { const }, \quad m, n=1,2,3, \ldots
$$

where:

$m$ and $n$ - are integers, often $m=n=1$, and

$\varphi_{x}(t)$ and $\varphi_{y}(t)$ - instantaneous phases of signals $x(t)$ and $y(t)$.

The phase synchronization index $\gamma_{H}$ is defined as (Mormann et al., 2000):

$$
\gamma_{H}=\left|\left\langle e^{i \varphi_{x y}(t)}\right\rangle_{t}\right|=\sqrt{\left\langle\cos \varphi_{x y}(t)\right\rangle_{t}^{2}+\left\langle\cos \varphi_{x y}(t)\right\rangle_{t}^{2}}
$$

where \langle\rangle is mean over time. If index $\gamma_{H}$ is zero, the phases will not be synchronized. Otherwise, if the value equals one, the phase difference will be constant. This index is sensitive to phases but independent of the amplitude of each signal.

Consider the Fourier spectrum of the analytic signal $x(t)+i \hat{x}(t)$ :

$$
Z(\omega)=X(\omega)+i \widehat{X}(\omega)=X(\omega)+i(-i \operatorname{sgn}(\omega)) X(\omega)=X(\omega)(1+\operatorname{sgn}(\omega))
$$

that is to say

$$
X(\omega)(1+\operatorname{sgn}(\omega))= \begin{cases}2 X(\omega) & \text { dla } \omega \geq 0 \\ 0 & \text { dla } \omega<0\end{cases}
$$

The Fourier spectrum of the analytic signal is a right-sided spectrum.

The phase synchronization calculated by means of the Hilbert transform has been widely used in the analysis of biomedical signals:

- EEG - electroencephalographic (Sun et al., 2012),

- ECG - electrocardiographic (Prokhorov et al., 2003),

- EMG - electromyographic (Wang et al., 2015),

- MMG - magnetomyographic (Govindan et al., 2015).

\section{Application in Uterine Bioelectrical Activity Signal Processing - Diagnosis of Preterm Delivery}

The data used in this study was recorded at the Department of Obstetrics and Gynaecology, Medical Centre, Ljubljana, between 1997 and 2006. These signals were provided as an open dataset on the Physionet web 

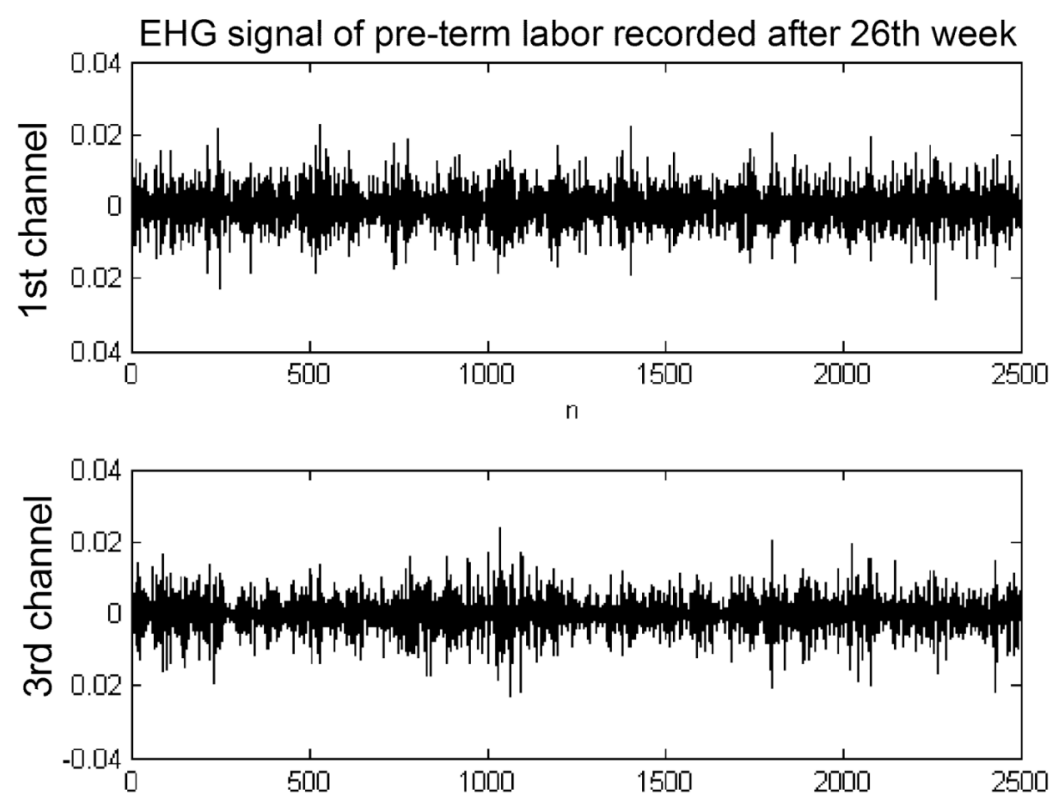

Figure 3. Example of EHG signal of a "prê-term delivery time series" recorded after the $26^{\text {th }}$ week: $1^{\text {st }}$ channel and $3^{\text {rd }}$ channel
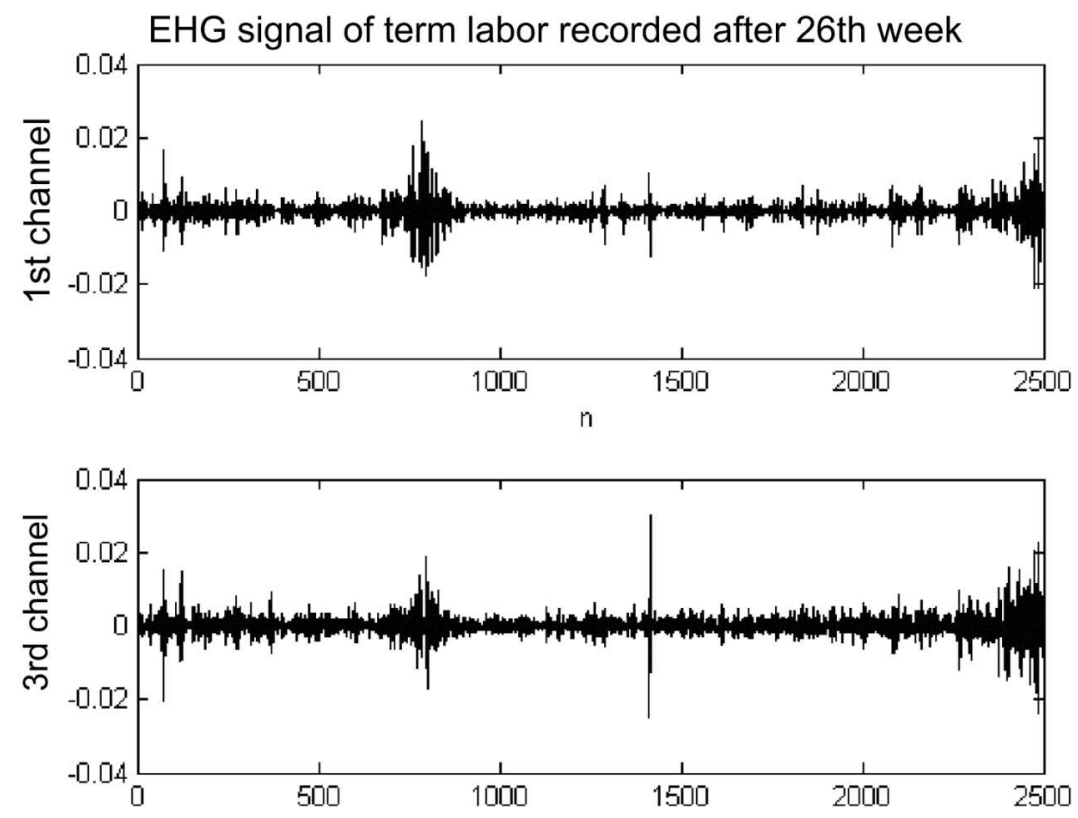

Figure 4. Example of EHG signal of a "têrm delivery time series" recorded after the $26^{\text {th }}$ week: $1^{\text {st }}$ channel and $3^{\text {rd }}$ channel 
Table 1. Values of synchronization indices $\gamma_{H}$ between the first and the third channels in the studied groups (according to the time of delivery and the time of recording, $*, * * p<0.001)$

\begin{tabular}{|c|c|c|c|c|c|}
\hline $\begin{array}{c}\text { Time of } \\
\text { recording }\end{array}$ & $\begin{array}{c}\text { PRE-TERM } \\
(\text { mean } \pm \mathrm{std})\end{array}$ & $\begin{array}{c}\text { TERM } \\
(\text { mean } \pm \mathrm{std})\end{array}$ & $\begin{array}{c}\text { Time of } \\
\text { recording }\end{array}$ & $\begin{array}{c}\text { PRE-TERM } \\
(\text { mean } \pm \mathrm{std})\end{array}$ & $\begin{array}{c}\text { TERM } \\
(\mathrm{mean} \pm \mathrm{std})\end{array}$ \\
\hline $\begin{array}{c}\text { Before the } \\
\text { 26th week }\end{array}$ & $\begin{array}{c}0.4050 \pm 0.188^{*} \\
(\mathrm{n}=15)\end{array}$ & $\begin{array}{c}0.5910 \pm 0.162^{*} \\
(\mathrm{n}=121)\end{array}$ & $\begin{array}{c}\text { After the } \\
26 \text { th week }\end{array}$ & $\begin{array}{c}0.387 \pm 0.154^{* *} \\
(\mathrm{n}=16)\end{array}$ & $\begin{array}{c}0.593 \pm 0.144^{* *} \\
(\mathrm{n}=91)\end{array}$ \\
\hline
\end{tabular}

(Goldberger et al., 2000). The EHG records, including the Term-Preterm Electrohysterogram Database, were collected from patients admitted to the hospital with the diagnosis of pre-term labor. One record per pregnancy was recorded. The records were 30 minutes long, had a sampling frequency $\left(f_{s}\right)$ of $20 \mathrm{~Hz}$ and consisted of three channels. The EHG signals were filtered by a $0.3 \mathrm{~Hz}-3 \mathrm{~Hz}$ band pass filter (Fele-Žorž et al., 2008). Groups of records were analyzed according to the time of delivery: term (Figure 3), pre-term (Figure 4), and according to the time of recording: before or after the $26^{\text {th }}$ week of gestation. Statistical analysis was performed by means of the non-parametric Mann-Whitney test. Values of synchronization index $\gamma_{H}$ between the first and the third channel in the studied groups (before or after the $26^{\text {th }}$ week of gestation) are presented in Table 1. Significant differences were found between the groups of records according to the time of delivery (term, pre-term) for all times of recording (before, after the $26^{\text {th }}$ week of gestation).

\section{Detection of Uterine Contractions from Electrohysterogram}

Changes in electrical potentials are associated with mechanical activity, i.e., contractions of uterine muscles. Uterine contraction (TOCO - signals) may be detected from electrohysterogram by several methods inter alia: filtration method, statistical method, higher-order zero crossing, and Hilbert envelope (Horoba et al., 2001; Karlsson et al., 2007; Radhakrishnan et al., 2000b). Figure 5 shows a raw EHG signal with marked areas of bioelectrical discharges (upper trace) and the corresponding uterine contractions - TOCO signal (lower trace). The curve representing mechanical activity was obtained by means of Hilbert envelope. 
Raw electrohysterographic signal with marked areas of bioelectrical discharges
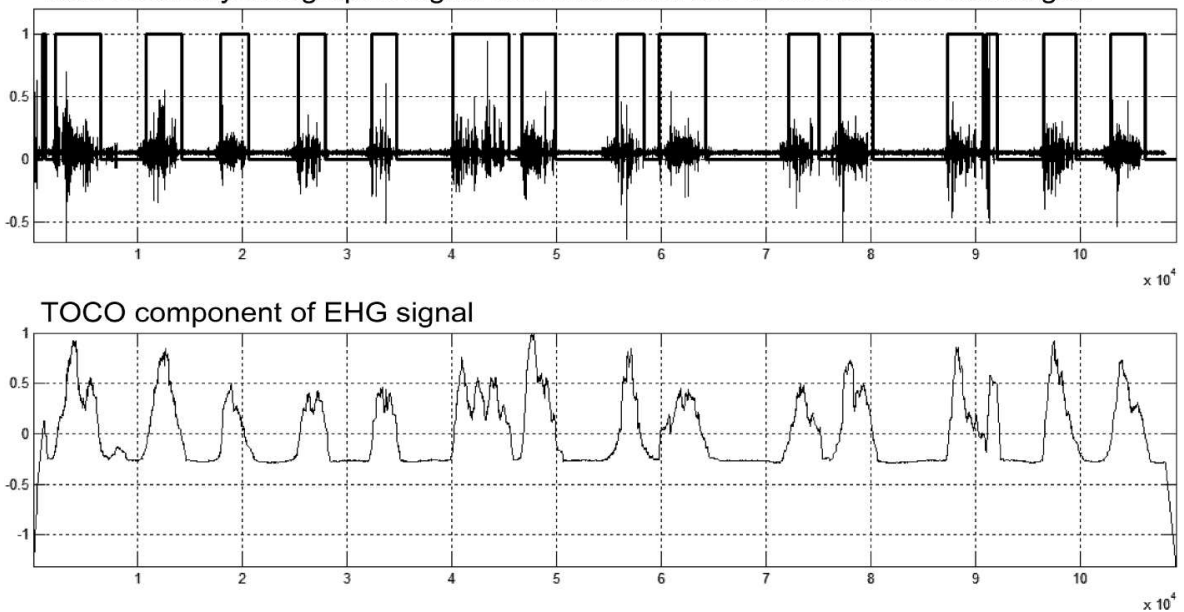

Figure 5. Raw EHG signal with marked areas of bioelectrical discharges (upper trace) and the corresponding uterine contractions TOCO signal (lower trace)

\section{Conclusions}

The above examples demonstrate the usefulness of the Hilbert transform in the study of uterine electrohysterographic signals. The use of this approach may provide diagnostically important information, valid in predicting preterm delivery.

\section{Acknowledgments}

This paper was supported by statutory funds S/WM/1/2014 from the Bialystok University of Technology.

\section{R E F E R E N C E S}

Boashash, B. (1992a). Estimating and interpreting the instantaneous frequency of a signal. I. Fundamentals. Proceedings of the IEEE, 80(4), 520-538.

Boashash, B. (1992b). Estimating and interpreting the instantaneous frequency of a signal. II. Algorithms and applications. Proceedings of the IEEE, 80(4), $540-568$.

Fele-Žorž, G., Kavšek, G., Novak-Antolič, Ž., \& Jager, F. (2008). A comparison of various linear and non-linear signal processing techniques to separate uterine EMG records of term and pre-term delivery groups. Medical \& Biological Engineering \& Computing, 46(9), 911-922. 
Gabor, D. (1946). Theory of communication. Part 1: The analysis of information. Journal of the Institution of Electrical Engineers-Part III: Radio and Communication Engineering, 93(26), 429-441.

Goldberger, A. L., Amaral, L. A., Glass, L., Hausdorff, J. M., Ivanov, P. C., Mark, R. G., Mietus, J. E., et al. (2000). Physiobank, physiotoolkit, and physionet components of a new research resource for complex physiologic signals. Circulation, 101(23), e215-e220.

Govindan, R. B., Siegel, E., Mckelvey, S., Murphy, P., Lowery, C. L., \& Eswaran, H. (2015). Tracking the Changes in Synchrony of the Electrophysiological Activity as the Uterus Approaches Labor Using Magnetomyographic Technique. Reproductive Sciences, 22 (5), 595-601.

Hahn, S. L. (1996). Hilbert transforms in signal processing. Boston, London: Artech House.

Horoba, K., Jezewski, J., Wrobel, J., \& Graczyk, S. (2001). Algorithm for detection of uterine contractions from electrohysterogram. Engineering in Medicine and Biology Society, 2001. Proceedings of the 23rd Annual International Conference of the IEEE, 3, 2161-2164. DOI:10.1109/IEMBS.2001.1017198

Karlsson, B., Terrien, J., Gudmundsson, V., Steingrimsdottir, T., \& Marque, C. (2007, January). Abdominal EHG on a 4 by 4 grid: mapping and presenting the propagation of uterine contractions. In T. Jarm, P. Kramar, \& A. Zupanic (Eds.), 11th Mediterranean Conference on Medical and Biomedical Engineering and Computing 2007 (pp. 139-143). Berlin, Heidelberg, Germany: Springer.

MATLAB Newsgroup (1997, June 19). Re: hilbert transform [Online forum comment] Retrieved from http://www.mathworks.com/matlabcentral/newsread er/view_thread/422

Mormann, F., Lehnertz, K., David, P., \& Elger, C. E. (2000). Mean phase coherence as a measure for phase synchronization and its application to the EEG of epilepsy patients. Physica D: Nonlinear Phenomena, 144(3), 358-369.

Nagarajan, R., Eswaran, H., Wilson, J. D., Murphy, P., Lowery, C., \& Preissl, H. (2003). Analysis of uterine contractions: a dynamical approach. The Journal of Maternal-Fetal \& Neonatal Medicine, 14(1), 8-21.

Oczeretko, E., Kitlas, A., Swiatecka, J., Borowska, M., \& Laudanski, T. (2005). Nonlinear dynamics in uterine contractions analysis. In G. A. Losa, D. Merlini, T. F. Nonnenmacher, \& E. R. Weilbel (Eds.), Fractals in Biology and Medicine (pp. 215-222). Basel, Switzerland: Birkhäuser.

Prokhorov, M. D., Ponomarenko, V. I., Gridnev, V. I., Bodrov, M. B., \& Bespyatov, A. B. (2003). Synchronization between main rhythmic processes in the human cardiovascular system. Physical Review E, 68(4), 041913.

Radhakrishnan, N., Wilson, J. D., Lowery, C., Murphy, P., \& Eswaran, H. (2000a). Testing for nonlinearity of the contraction segments in uterine electromyography. International Journal of Bifurcation and Chaos, 10(12), 2785-2790. 
Radhakrishnan, N., Wilson, J. D., Lowery, C., Eswaran, H., \& Murphy, P. (2000b). A fast algorithm for detecting contractions in uterine electromyography. Engineering in Medicine and Biology Magazine, IEEE, 19(2), 89-94.

Sun, J., Hong, X., \& Tong, S. (2012). Phase synchronization analysis of EEG signals: an evaluation based on surrogate tests. IEEE Transactions on Biomedical Engineering, 59(8), 2254-2263.

Sahoo, J. P., Behera, S., \& Ari, S. (2011). A Novel Technique for QRS Complex detection in ECG Signal based on Hilbert Transform and Autocorrelation. International Conference on Electronics Systems (ICES-2011).

Toland, J. F. (1997). A few remarks about the Hilbert transform. Journal of Functional Analysis, 145(1), 151-174.

Wang, L., Lu, A., Zhang, S., Niu, W., Zheng, F., \& Gong, M. (2015). Fatigue-related electromyographic coherence and phase synchronization analysis between antagonistic elbow muscles. Experimental Brain Research, 233(3), 971-982. 